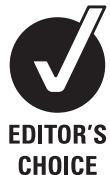

CHOICE

\title{
Development of diastolic heart failure in a 6-year follow-up study in patients after acute myocarditis
}

\author{
Felicitas Escher, ${ }^{1}$ Dirk Westermann, ${ }^{1}$ Regina Gaub, ${ }^{1}$ Johannes Pronk, ${ }^{1}$ Thomas Bock, ${ }^{2}$ \\ Nidal Al-Saadi, ${ }^{3}$ Uwe Kühl, ${ }_{1}^{1}$ Heinz-Peter Schultheiss, ${ }^{1}$ Carsten Tschöpe ${ }^{1,4}$
}

\section{See Editorial, p 685 \\ ${ }^{1}$ Department of Cardiology and Pneumonology, \\ Charité-University Medicine \\ Berlin, Campus Benjamin \\ Franklin, Berlin, Germany \\ ${ }^{2}$ Robert Koch Institute Berlin, \\ Berlin, Germany \\ ${ }^{3}$ Interdisciplinary Cardiac Imaging Center Berlin, Berlin, Germany \\ ${ }^{4}$ Berlin-Brandenburg Center for Regenerative Therapies, Berlin Germany}

\section{Correspondence to}

C Tschöpe, Department of

Cardiology and Pneumonology, Charité-Universitätsmedizin

Berlin, Campus Benjamin

Franklin, Hindenburgdamm 30,

D-12200 Berlin, Germany;

carsten.tschoepe@charite.de

Accepted 14 September 2010 Published Online First

5 December 2010

\section{ABSTRACT \\ Background The aim of this study was to analyse the long-term prognosis of patients with acute myocarditis (AMC) who had been discharged from hospital while having normal left ventricular (LV) function.}

Methods and results 50 patients with acute myocarditis who underwent endomyocardial biopsies (EMBs) were prospectively studied. Their clinical condition was examined during a mean follow-up period of 72 (54-78) months, including tissue Doppler imaging (TDI). 4\% (2/50) died, and 6\% (3/50) developed dilated cardiomyopathy. $45 / 50$ (90\%) showed a normal or improvement in LV function over time. In the course of the follow-up, 49\% (22/45) suffered from heart failure symptoms despite a normal ejection fraction (HFNEF). This was associated with an abnormal E/A ratio, an impaired deceleration time of early mitral flow velocity and isovolumic relaxation time, and a pathological increase in the LV filling index $E / E^{\prime}$, in contrast to patients without heart failure symptoms (E/E ${ }^{\prime}$ septal 10.9 (9.3-13.8) vs $6.8(6.4-9.1) ; p=0.001)$. Plasma $\mathrm{N}$-terminal proB-type natriuretic peptide levels were increased threefold in patients with HFNEF (19.9 $(10.6-24.1)$ vs $7.3(4.2-11.9) \mathrm{pmol} / \mathrm{l} ; \mathrm{p}=0.006)$.

Conclusions It is assumed that the evidence for AMC is associated not only with the risk of developing LV dilatation but also with an increased risk of symptomatic diastolic dysfunction after several years.

\section{INTRODUCTION}

Severe acute myocarditis (AMC) is a serious disorder caused by cardiotropic viral infection. ${ }^{12}$ The clinical course and presentation are highly variable. Currently, there are no apparent clinical criteria which reliably predict which patients with AMC are likely to recover. In the natural history of AMC, the transition to a chronically progressive disease with progressive impairment of left ventricular (LV) function-consistent with the clinical picture of dilated cardiomyopathy (DCM) - is seen in 20\% of patients. This is often linked to chronic inflammation and viral persistence due to an inadequate immune response after AMC. The affected patients have an increased risk of cardiac death or the need for cardiac transplantation. ${ }^{3-5}$

However, the majority of patients do not develop significant LV dilatation and severe deterioration of the ejection fraction (EF) over time. In general, 80\% of patients with AMC are discharged from hospital with normal cardiac function and are considered to have been cured. Medical treatment and examination are usually discontinued after several months. However, the question as to whether or not they recover fully over time or whether their morbidity is still compromised after years has not yet been clarified. Thus, definitive data of patients with $\mathrm{AMC}$ are lacking concerning the prediction of the long-term clinical prognosis and the identification of distinct aetiopathogenetic subsets based on endomyocardial biopsies (EMBs).

Therefore, we investigated the long-term followup and prognosis of patients after AMC.

\section{METHODS}

\section{Patients}

The study subjects consisted of 50 consecutive patients (37 males, 13 females) admitted to our institute from January 1995 to November 2004. In detail, the tentative, clinically suspected diagnosis of AMC was ascertained in patients who had presented with a very recent onset of congestive heart failure symptoms, with an abrupt onset of complaints such as angina or dyspnoea, and any infarct-like presentation such as elevated serum markers of myocardial injury (troponin $\mathrm{T}$ and creatine kinase/ creatine kinase-MB) and/or newly developed ECG changes (ST-segment elevation or Twave inversion). No patient was included with previously known cardiomyopathy or a family history of cardiomyopathy. Exclusion criteria were antiviral, immunmodulatory or immunosuppressive therapy within the last 6 months, clinical or biochemical evidence for the presence of concomitant chronic inflammatory disease, chronic renal insufficiency (creatinine $\geq 1.4 \mathrm{mmol} / \mathrm{l}$ ), inability to understand the consent form, participation in or consent to participate in another study, or malignant disease. The demographic and clinical characteristics of patients at baseline are summarised in table 1. All patients underwent heart catheterisation for the evaluation of their coronary status. After angiographic exclusion of coronary artery disease and other possible causes of LV dysfunction EMBs from the right ventricular septum were obtained using a flexible bioptome (Westmed, St Ingbert, Germany) via the femoral vein approach. ${ }^{6-8}$ According to the recently published statement of the American College of Cardiology (ACC), American Heart Association (AHA) and European Society for Cardiology (ESC), EMB should be performed in the setting of unexplained, new-onset heart failure of $<2$ weeks duration associated with a normal-sized or dilated left ventricle in addition to haemodynamic compromise (Class of Recommendation I, Level of Evidence B). All patients were invited to participate in a follow-up at a dedicated outpatient clinic. Assessment included physical examination, analysis of plasma N-terminal (NT)-proB-type natriuretic peptide (NT-proBNP) 
Table 1 Patients' characteristics at baseline and follow-up

\begin{tabular}{|c|c|c|c|c|}
\hline Demographics & $\begin{array}{l}\text { All at } \\
\text { baseline }\end{array}$ & $\begin{array}{l}\text { Follow-up } \\
\text { patients } \\
\text { without HFNEF }\end{array}$ & $\begin{array}{l}\text { Follow-up } \\
\text { patients } \\
\text { with HFNEF }\end{array}$ & p Value \\
\hline Number of patients & 50 & 23 & 22 & NS \\
\hline Gender, male/female & $37 / 13$ & $20 / 3$ & $14 / 8$ & NS \\
\hline $\begin{array}{l}\text { Follow-up period, } \\
\text { months }\end{array}$ & 72 & $72(51-76)$ & $71(55-80)$ & NS \\
\hline NYHA & $38 \mathrm{II} / 3 \mathrm{III} / 1 \mathrm{IV}$ & 231 & $17 \mathrm{II} / 5 \mathrm{III}$ & $<0.001$ \\
\hline $\begin{array}{l}\text { Hospitalisation } \\
\text { with } \mathrm{HF}, \mathrm{n}\end{array}$ & 42 & 1 & 7 & NS \\
\hline Days in hospital & & 4 & $7.0(4.0-8.0)$ & NS \\
\hline NT-proBNP pmol// & $29.2(10.2-49.8)$ & $7.3(4.2-11.9)$ & $19.9(10.6-24.1)$ & 0.006 \\
\hline \multicolumn{5}{|l|}{ Medications, n } \\
\hline$\beta$-Blockers & 12 & 1 & 2 & NS \\
\hline RAAS inhibitors & 21 & 1 & 4 & NS \\
\hline Diuretics & 11 & 0 & 0 & NS \\
\hline
\end{tabular}

$\mathrm{p}<0.05$ follow-up patients without HFNEF versus follow-up patients with HFNEF Values are expressed as the median (25-75\% quartile).

HFNEF, heart failure with a normal ejection fraction; NS, non-significant; NT-proBNP, $\mathrm{N}$-terminal pro-brain natriuretic peptide; NYHA, New York Heart Association; RAAS, renin-angiotensin-aldosterone system.

and high sensitive C-reactive protein (hsCRP) levels and transthoracic echocardiography at each visit. Patients with severe arterial hypertension, valvular heart disease, metabolic and endocrine diseases were excluded from the study.

\section{Definition and assessment of ventricular function}

Conventional Doppler flow velocities were recorded in the apical four-chamber view obtained by a Vivid 7 (GE Healthcare, Chalfont St Giles, UK) as described previously. ${ }^{9}$ The diagnosis of heart failure with a normal ejection fraction (HFNEF) was defined according to the recommendation of the Heart Failure and Echocardiography Associations of the ESC. ${ }^{10}$ Three obligatory conditions need to be satisfied for the diagnosis of HFNEF: (1) presence of symptoms of heart failure; (2) presence of normal LV systolic function; and (3) evidence of diastolic LV dysfunction. The ratio of the transmitral flow velocity to annular velocity $\mathrm{E} / \mathrm{E}^{\prime}$ is considered diagnostic evidence of the presence of diastolic LV dysfunction ranging from 8 to 15 in addition to bloodflow Doppler measurements and increased levels of the biomarker NT-proBNP. Measurements of chamber dimensions, including LV end-diastolic diameter, septal and posterior wall thicknesses, and left atrial size, were evaluated according to standard procedures. The EF was measured by the biplane Simpson method. Mitral inflow measurements included peak early (E) and peak late (A) flow velocities, the E/A ratio, the deceleration time of early mitral flow velocity and the isovolumic relaxation time. Data were adjusted for age and heart rate according to the pertinent guidelines. ${ }^{11}$ Tissue Doppler imaging (TDI) of the mitral annulus movement was obtained from the apical four-chamber view. A $1.5 \mathrm{~mm}$ sample volume was placed sequentially at the lateral and septal annular sites. Analysis was performed by pulsed wave Doppler for the systolic $\left(S^{\prime}\right)$ and the early $\left(E^{\prime}\right)$ and late $\left(A^{\prime}\right)$ diastolic peak velocities. Overall diastolic stage, as determined from the pattern of mitral and pulmonary venous flow, was defined as impaired relaxation: E/A ratio $<1$, deceleration time $>220 \mathrm{~ms}$. The LV filling index was not evaluated at the beginning of the study, since only recently have guidelines indicated the importance of tissue Doppler for evaluating diastolic function. ${ }^{10} 11$

\section{Detection of viral genomes in EMBs by nested PCR (nPCR) and} reverse transcription-PCR (RT-PCR)

Four specimens were subjected to molecular biological investigation of cardiotropic viral genomes according to published techniques. $^{2}$ In brief, nPCR/RT-PCR was performed on RNA extracted from EMBs for enteroviruses (EVs)-including coxsackie viruses and echoviruses-and on DNA for Epstein-Barr virus (EBV), parvovirus B19 (B19) and human herpes virus 6 (HHV6). As a control for the successful extraction of DNA and RNA from heart muscle tissue, oligonucleotide sequences were chosen from the DNA sequence of the glyceraldehyde 3-phosphate dehydrogenase gene. The specificity of all amplification products was confirmed by automatic DNA sequencing. ${ }^{12}$

\section{Immunohistochemical staining}

EMBs were obtained from the right ventricular septum, frozen in liquid nitrogen and stored at $-80^{\circ} \mathrm{C}$. For immunohistological evaluation, specimens were embedded in Tissue Tec (SLEE, Mainz, Germany) and immediately snap-frozen in methylbutane which had been cooled in liquid nitrogen, and then stored again at $-80^{\circ} \mathrm{C}$ until processing. Embedded specimens were cut serially into cryosections of $5 \mu \mathrm{m}$ thickness and placed on $10 \%$ poly-L-lysine-precoated slides. Immunohistochemistry was used for the characterisation of inflammatory infiltrates. Myocardial inflammation was defined by the detection of infiltrating lymphocytes (median cell count $>7.0 \mathrm{cells} / \mathrm{mm}^{2}$ ) and macrophages (median cell count $>35.0$ cells $/ \mathrm{mm}^{2}$ ) in association with enhanced expression of intercellular cell adhesion molecule-1 (ICAM-1) expressed on interstitial or endothelial cells. Histological diagnosis was based on the Dallas criteria. ${ }^{13}$ The coded slides were examined in a blinded fashion. Immunohistological staining was carried out and the immunoreactivity was quantified by digital image analysis (unit:area fraction) at 200-fold magnification as described in detail elsewhere. ${ }^{14} 15$

\section{Cardiac MRI (CMR)}

CMR was used in a subgroup of patients according to the CMR protocol of Gutberlet et al. ${ }^{16}$ CMR studies were performed in a $1.5 \mathrm{~T}$ system (GE Healthcare, Milwaukee, Wisconsin, USA). Intravascular injection of gadolinium-diethylenetriaminepentaacetate (Magnevist, Schering, Germany) was used, to determine late enhancement.

\section{Statistical analysis and explanation of statistical tables}

Data are shown as median values (25-75\% percentile). After having established that the data were not distributed normally, the non-parametric Mann-Whitney $U$ test was used. The Fisher exact test was used to analyse categorical variables. A probability value of $<0.05$ was considered statistically significant. Data were analysed with Graphpad 5.01 (PRISM, San Diego, California, USA). The authors had full access to the data and take responsibility for its integrity.

\section{RESULTS}

\section{Demographic and clinical data}

Baseline population and clinical characteristics are presented in table 1 . The study included 50 patients, 13 women and 37 men, with a mean age of 43.5 years (range $35.0-57.2$ years). The entire cohort presented with chest pain, ST elevation and cardiac biomarker changes, and proceeded to coronary angiography during the same admission. The mean follow-up period was 72 months (range 54-78). Two patients developed a dilated cardiomyopathy and two patients died due to cardiovascular events within 2 years after their first presentation. One patient (a 42-year-old male) required cardiac transplantation 1.5 years after presentation. At baseline, the majority of patients were in New York Heart Association (NYHA) class II. During follow-up, 
22 of the 45 patients had clinical signs of heart failure with normal LV systolic function (HFNEF), and dimensions as shown in table 2. Eight of these patients developed a worse NYHA functional status than that noted at the time of presentation. No difference was seen between patients with or without HFNEF with respect to age. Plasma NT-proBNP levels were increased threefold in patients with HFNEF when compared with patients considered to have normal LV diastolic function without symptoms during the follow-up. HsCRP was increased in the acute phase (mean $9.1 \pm 3.2 \mathrm{mg} / \mathrm{l}$ ), but did not differ between the two groups of patients with later HFNEF or without HFNEF. At follow-up, in no patient was an increased value of hsCRP (mean $3.2 \pm 0.5 \mathrm{mg} / \mathrm{l}$ ) seen.

\section{Detection of viral genomes and immunohistological staining in EMBs at presentation}

The frequencies of viral genomes detected by nPCR are shown in table 3. Mean virus load was calculated for B19 with $279 \pm 202$ genome equlivalence per microgram (ge), for HHV6 with $247 \pm 98$ ge, for EBV with 59 ge and for EV with $143 \pm 67$ ge. The prevalence of virus genomes and virus load was not found to be significantly increased in patients with HFNEF when compared with patients without any evidence of HFNEF (16/22 vs 12/23 $\mathrm{p}<0.99$ ). With regard to the immunohistological staining, neither T lymphocytic or macrophage infiltrates nor endothelial expression of cell adhesion molecules were distinguished between viruspositive and virus-negative patients, or between patients with and without HFNEF. However, the numbers of lymphocytes and macrophages were increased $\left(>7\right.$ cells $\left./ \mathrm{mm}^{2}\right)$ in both virus-positive and virus-negative tissues, and adhesion molecule expression correlated with infiltrating inflammatory cell numbers.

\section{Heart dimensions}

Echocardiographic measurements at baseline and at follow-up are shown in tables 2 and 4 . The mean LV diastolic dimension as well as the left atrial diameter were not elevated at baseline and did not change in patients with or without HFNEF at follow-up.

\section{Systolic function of the left ventricle}

In patients with or without HFNEF, no initial difference in the mean LV function was seen as measured by the EF. Three of the 23 patients without heart failure symptoms and three of the 22 patients with HFNEF recovered from impaired LV systolic function $(\mathrm{EF}<50 \%)$ and showed a complete normalisation of LV

Table 2 Conventional echocardiography at baseline

\begin{tabular}{lc}
\hline Echocardiography parameters & No. at baseline \\
\hline Echocardiography dimensions & \\
LVEF, Simpson (\%) & $67.0(53.5-73.5)$ \\
LA parasternal, mm & $36.0(34.0-38.0)$ \\
LVEDD, mm & $50.0(48.0-55.5)$ \\
LVESD, mm & $33.0(29.5-37.5)$ \\
Septum, mm & $10.0(10.0-12.0)$ \\
Posterior wall, mm & $10.0(9.0-11.0)$ \\
Diastolic indices, conventional & \\
E, m/s & $0.77(0.61-0.89)$ \\
A, m/s & $0.69(0.59-0.80)$ \\
E/A & $1.1(0.83-1.5)$ \\
DT, ms & $183.0(180.0-199.5)$ \\
IVRT, ms & $93.0(88.8-112.0)$
\end{tabular}

Values are expressed as the median $(25-75 \%$ quartile).

$D T$, deceleration time; $E / A$, ratio of peak early and late mitral flow;

IVRT, isovolumic relaxation time; LA, left atrium; LVEDD, left ventricular end-diastolic diameter; LVEF, left ventricular ejection fraction; LVESD, left ventricular end-systolic diameter.
Table 3 Distribution of virus genomes by PCR and intramyocardial inflammation by immunohistochemistry in patients with and without HFNEF

\begin{tabular}{lll}
\hline & $\begin{array}{l}\text { Patients without } \\
\text { HFNEF }\end{array}$ & $\begin{array}{l}\text { Patients with } \\
\text { HFNEF }\end{array}$ \\
\hline $\mathrm{B} 19, \mathrm{n}$ & 5 & 12 \\
$\mathrm{EV}, \mathrm{n}$ & 2 & 0 \\
$\mathrm{HHV6}, \mathrm{n}$ & 1 & 4 \\
$\mathrm{EBV}, \mathrm{n}$ & 0 & 0 \\
$\mathrm{~B} 19+\mathrm{EBV}, \mathrm{n}$ & 1 & 0 \\
B19+HHV6+EBV, $\mathrm{n}$ & 3 & 0 \\
Intramyocardial inflammation & & \\
ICAM-1 expression, $\mathrm{n}$ & 23 & 22 \\
CD3 $^{+}$lymphocytes $/ \mathrm{mm}^{2}$ & $18.8(10.5-24.2)$ & $17.6(11.6-34.2)$ \\
${\text { Cytotoxic T lymphocytes } / \mathrm{mm}^{2}}^{\text {Mac-1 (macrophages } / \mathrm{mm}^{2}}$ & $2.5(1.2-6.3)$ & $4.5(3.5-7.9)$ \\
\hline
\end{tabular}

B19, parvovirus B19; EBV, Epstein-Barr virus; EV, enterovirus; HFNEF, heart failure with a normal ejection fraction; HHV6, human herpes virus 6; ICAM-1, intercellular cell adhesion molecule-1.

function during the follow-up. Two of the patients with an initial mildly reduced EF developed dilated cardiomyopathy.

Diastolic function of the left ventricle: conventional Doppler and TDI echocardiography at follow-up

Table 4 presents diastolic indices provided by TDI. Analysis of diastolic filling patterns at the beginning of our study indicated

Table 4 Echocardiography dimensions and diastolic indices of conventional Doppler and tissue Doppler imaging echocardiography at follow-up

\begin{tabular}{|c|c|c|c|}
\hline Parameters & $\begin{array}{l}\text { Follow-up } \\
\text { patients } \\
\text { without HFNEF }\end{array}$ & $\begin{array}{l}\text { Follow-up } \\
\text { patients } \\
\text { with HFNEF }\end{array}$ & p Value \\
\hline \multicolumn{4}{|l|}{ Heart dimensions } \\
\hline LVEF, Simpson (\%) & $64.0(63.0-68.0)$ & $64.0(59.0-68.0)$ & 0.42 \\
\hline LA parasternal, mm & $38.0(37.0-40.0)$ & $36.5(33.0-42.0)$ & 0.33 \\
\hline LVEDD, mm & $52.0(49.0-55.0)$ & $49.0(45.0-52.0)$ & 0.05 \\
\hline LVESD, mm & $32.0(27.0-35.0)$ & $31.0(27.0-33.5)$ & 0.48 \\
\hline Septum, mm & $11.0(10.0-11.0)$ & $10.0(9.0-11.5)$ & 0.57 \\
\hline Posterior wall, mm & $11.0(10.0-11.0)$ & $11.0(9.0-11.0)$ & 0.57 \\
\hline LVOT, m/s & $1.0(0.92-1.10)$ & $1.0(0.95-1.13)$ & 0.79 \\
\hline \multicolumn{4}{|l|}{ Conventional Doppler } \\
\hline $\mathrm{E}, \mathrm{m} / \mathrm{s}$ & $0.7(0.63-0.84)$ & $0.81(0.71-0.94)$ & 0.16 \\
\hline$A, \mathrm{~m} / \mathrm{s}$ & $0.61(0.47-0.7)$ & $0.84(0.74-0.99)$ & 0.001 \\
\hline$E / A$ & $1.12(1.03-1.42)$ & $0.97(0.81-1.18)$ & 0.012 \\
\hline IVRT, ms & $85.6(73.9-95.6)$ & $102.7(91.3-107.9)$ & 0.0024 \\
\hline DT, ms & $205(180-216)$ & $241(205-255)$ & 0.02 \\
\hline \multicolumn{4}{|l|}{ Tissue Doppler } \\
\hline $\mathrm{S}_{\text {lateral, }} \mathrm{m} / \mathrm{s}$ & $0.08(0.06-0.09)$ & $0.07(0.05-0.08)$ & 0.09 \\
\hline $\mathrm{E}_{\text {lateral, }} \mathrm{m} / \mathrm{s}$ & $0.09(0.08-0.11)$ & $0.07(0.06-0.08)$ & 0.006 \\
\hline $\mathrm{A}_{\text {lateral, }}^{\prime} \mathrm{m} / \mathrm{s}$ & $0.07(0.05-0.09)$ & $0.07(0.06-0.08)$ & 0.9 \\
\hline$E^{\prime} / A_{\text {lateral }}^{\prime}$ & $1.43(0.87-1.95)$ & $0.92(0.61-1.3)$ & 0.03 \\
\hline $\mathrm{E} / \mathrm{E}_{\text {lateral }}^{\prime}$ & $7.9(6.9-8.9)$ & $9.4(8.3-16.9)$ & 0.006 \\
\hline $\mathrm{S}_{\text {septal, }}^{\prime} \mathrm{m} / \mathrm{s}$ & $0.06(0.06-0.07)$ & $0.06(0.05-0.06)$ & 0.07 \\
\hline $\mathrm{E}_{\text {septal, }}^{\prime} \mathrm{m} / \mathrm{s}$ & $0.09(0.08-0.11)$ & $0.07(0.06-0.09)$ & 0.03 \\
\hline $\mathrm{A}_{\text {septal, }}^{\prime} \mathrm{m} / \mathrm{s}$ & $0.07(0.06-0.09)$ & $0.07(0.07-0.08)$ & 0.9 \\
\hline$E^{\prime} / A_{\text {septal }}^{\prime}$ & $1.19(0.98-1.45)$ & $0.9(0.75-1.25)$ & 0.04 \\
\hline$E / E_{\text {septal }}^{\prime}$ & $6.8(6.4-9.1)$ & $10.9(9.3-13.8)$ & 0.001 \\
\hline$E / E_{\text {mean }}^{\prime}$ & $7.4(6.8-9.8)$ & $10.8(9.5-14.4)$ & 0.001 \\
\hline
\end{tabular}

Values are expressed as the median (25-75\% quartile).

$A^{\prime}$, late diastolic velocity; $D T$, deceleration time; $E^{\prime}$, early diastolic peak velocity; $E / A$, ratio of peak early and late mitral flow; $E / E^{\prime}$, ratio of transmitral flow velocity to annular velocity; HFNEF, heart failure with a normal ejection fraction; IVRT, isovolumic relaxation time; LA, left atrium; LVEDD, left ventricular end-diastolic diameter; LVEF, left ventricular ejection fraction; LVESD, left ventricular end-systolic diameter; LVOT, left ventricular outflow tract; $S^{\prime}$, systolic peak velocity 
a) EF (\%) at baseline and follow-up in asymptomatic patients

ejection fraction $(\%)$

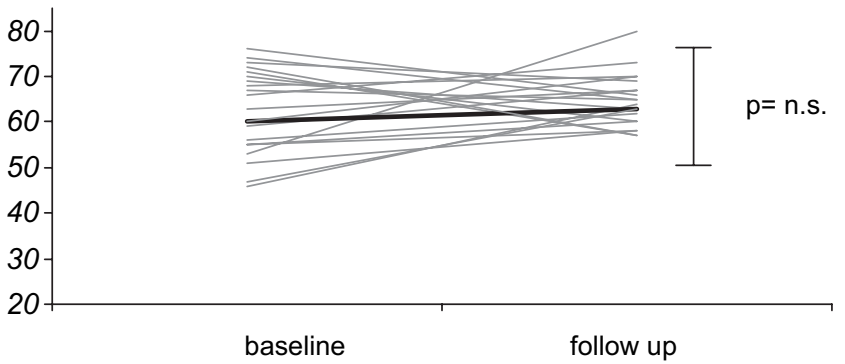

b) EF (\%) at baseline and follow-up in symptomatic patients ejection fraction (\%)

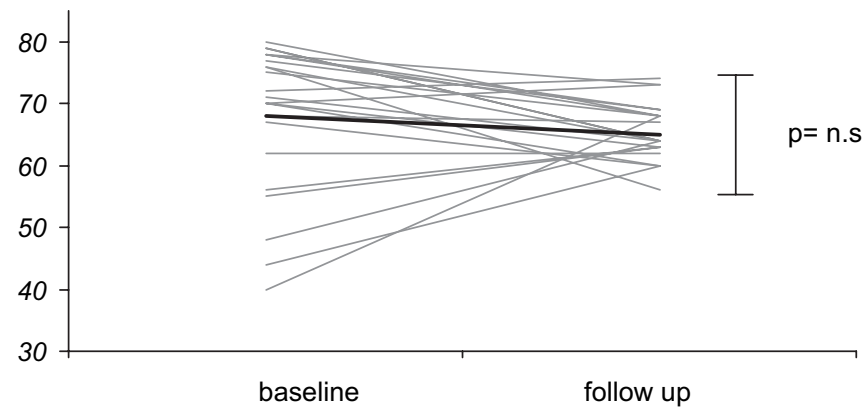

Figure 1 Haemodynamic courses as measured by conventional echocardiography using ejection fraction (\%) in (A) asymptomatic patients and (B) symptomatic patients.

no diastolic dysfunction. At baseline, there were no differences between the patients who were classified as being with or without HFNEF during the follow-up. During the follow-up, patients with HFNEF had a significantly prolonged isovolumic relaxation time and a significantly reduced $\mathrm{E} / \mathrm{A}$ ratio in comparison with patients without HFNEF (figure 1). By examining the filling index, $\mathrm{E} / \mathrm{E}^{\prime}$, we were able to observe a significant increase in this parameter in patients with HFNEF (table 4).

\section{Hospitalisation rate}

During the observation period, $32 \%(7 / 22)$ of patients with HFNEF were hospitalised after heart failure symptomatology and had a median length of stay of 7.0 (4.0-8.0) days in hospital during readmission, whereas only one patient without HFNEF was initially in hospital for 4 days (table 1). a)

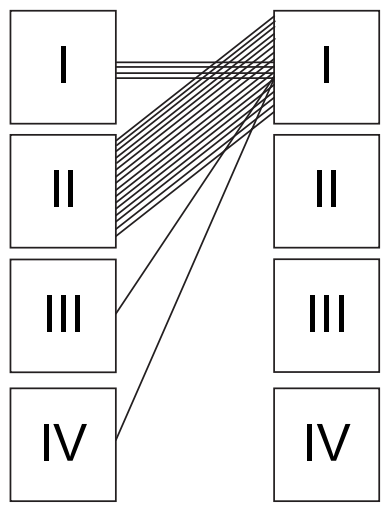

baseline b)

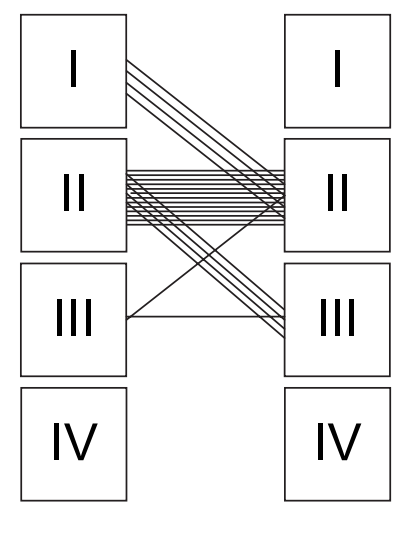

Figure 3 Changes of New York Heart Association functional class during baseline and follow-up after 6 years in (A) $n=23$ patients without HFNEF (heart failure with a normal ejection fraction) and (B) $n=22$ patients with HFNEF.

\section{CMR results}

In 17 patients ( $n=12$ with HFNEF and $n=5$ without HFNEF), CMR results were available. In none of the CMRs were signs of acute myocarditis detectable. In all of the CMRs of patients with HFNEF, late gadolinium enhancement (LGE) was detectable, whereas only one patient without HFNEF showed minimal signs of LGE. Representative images are shown in figure 2.

\section{DISCUSSION}

The salient finding of the current study is that after AMC, $90 \%$ $(45 / 50)$ of patients recover early with regard to EF. Therefore, they do not remain under strict control and medical treatment is often withdrawn. However, 4-6 years after AMC-despite a normal EF-49\% (22/45) of the patients in this study showed heart failure symptomatology present with dyspnoea and exercise intolerance, resulting in a significant NYHA status of II-III (figure 3) and increased BNP levels. This was associated with diastolic dysfunction. Typical risk factors for HFNEF such as severe hypertension and diabetes mellitus had been excluded. In general it has been thought ${ }^{17}$ that in cases where AMC had been acquired from a natural cause, the majority of patients recover completely over the course of time due to EF and LV dimensions. The data presented here show for the first time that an important number of these patients develop HFNEF with diastolic dysfunction. At the beginning of our study, we investigated 50 patients with AMC who were referred to our hospital with acute

Figure 2 Representative examples of cardiovascular MRIs of $(A)$ patients without HFNEF (heart failure with a normal ejection fraction) and (B) patients with HFNEF. (A) No evidence for late gadolinium enhancement; (B) subepicardial area of late gadolinium enhancement.
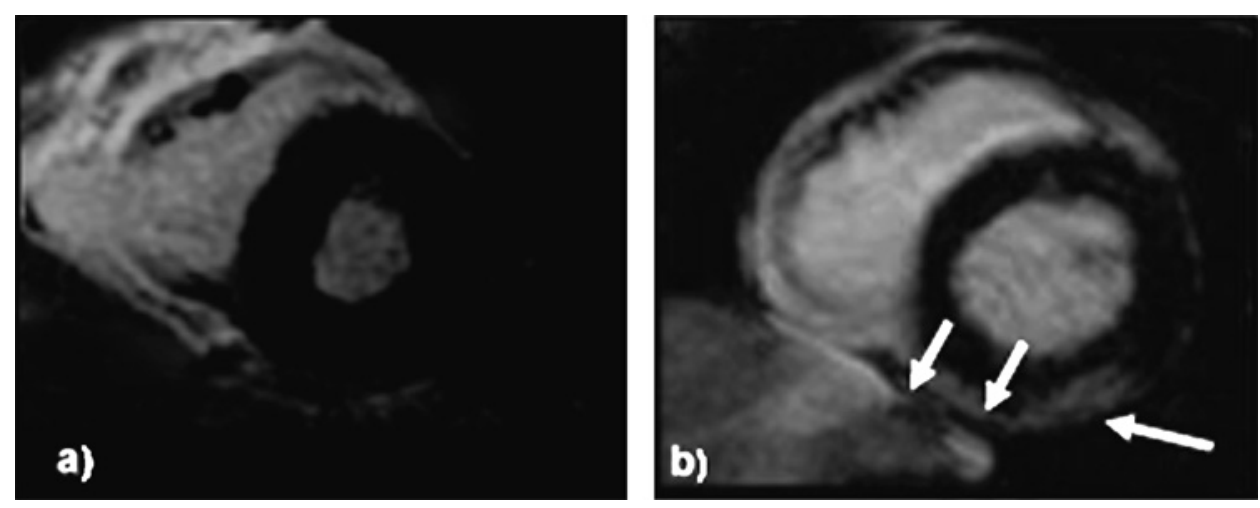
onset of severe heart failure symptoms. In all patients with acute myocardial damage as evidenced by increased creatine kinase and troponin $\mathrm{T}$ levels, coronary heart disease and myocardial infarction were ruled out by angiography. Active myocarditis based on the Dallas criteria was demonstrated in $\sim 40 \%$, and cardiac viral persistence was shown to be present in $\sim 70 \%$ of the patients' EMBs. Immunohistological signs of inflammation were shown in all patients. Our study confirms the finding that AMC subsequently leads to dilated cardiomyopathy and death in $~ 10-20 \%$ of patients. In accordance with recently published data, we observed the development of DCM in two of these patients in the follow-up period ${ }^{18}$; in one of the patients, cardiac transplantation was performed due to progression of heart failure and DCM. Furthermore, in agreement with the highly increased mortality rate described, two of the patients died of cardiac failure after AMC.

The clinical symptomatology was further verified by ascertainment of increased NT-proBNP levels, which can be deemed to be an objective marker for clinical symptoms in patients with heart failure including patients with HFNEF. ${ }^{19-21}$ At the same time, we have shown in the current study that the mitral flow pattern and LV filling index E/E'were pathologically increased in those patients who were still complaining about heart failure symptoms despite a normal EF. This is in contrast to patients without heart failure symptoms.

To date, the pathophysiological mechanisms leading to this heart failure symptomatology are still under debate. Diastolic dysfunction is known to be one key mechanism for the development of HFNEF and can occur in viral cardiomyopathy. Since diastolic function as measured by conventional blood flow Doppler was not impaired differently at baseline, we suggest that diastolic dysfunction had been acquired by patients with HFNEF in the interim, a fate which may have been initiated by inflammatory processes. As shown for ageing, female gender, hypertension, coronary heart disease, diabetes mellitus and obesity, $^{22}{ }^{23}$ our findings suggest that AMC is clearly associated with the development of diastolic dysfunction over a longer period of time, and therefore AMC can be a cause of heart failure symptomatology. We previously described a strong causal relationship between B19-induced inflammatory cardiomyopathy and HFNEF which is based on the background of an isolated diastolic dysfunction. ${ }^{7}$ However, in the present study at baseline, viral genomes did not differ significantly between patients with or without HFNEF. Therefore, it may be claimed that viral genomes do not necessarily predict HFNEF after AMC.

According to a recent publication of Kindermann et al, ${ }^{24}$ the quality and quantity of inflammation could be a risk factor for the development of HFNEF after AMC. Although the initial inflammation response did not differ between the patients investigated, a prolonged duration of inflammation even at a lower level could lead to a significant difference in the outcome. Ethically we were not allowed to take rebiopsies from the study group. Taking into account that one limitation of our study was that only a subgroup of $n=17$ patients was examined by CMR, in all explored HFNEF patients LGE was detectable, whereas only one patient without HFNEF showed LGE. Since late enhancement indicates zones of myocyte necrosis and fibrosis, we suggest that changes in the level of fibrosis may contribute to the development in HFNEF after AMC. Accumulation of cardiac fibrosis in the absence of LV hypertrophy is one of the well-known hallmarks of experimental viral myocarditis. ${ }^{25}$ Necrosis of adjacent myocytes may be the consequence of persistent inflammatory processes of the myocardium due to infiltration of immune cells. Diastolic dysfunction, however, is the sum of a complex sequence of multiple interrelated events, including prolonged ventricular relaxation, loss of ventricular suction, increased myocardial fibrosis, increased chamber stiffness and endothelial dysfunction, all of which result in symptoms of angina, dyspnoea and exercise limitation. ${ }^{26-28}$ In those patients with a spontaneous recovery of the disease with regard to EF, treatment with inhibition of neurohumoral stimulation was terminated due to the lack of long-term follow-up data. This may lead to a slow but progressive profibrotic process in patients subsequent to AMC, a matter which is associated with the development of diastolic dysfunction.

Moreover, in line with other studies showing that HFNEF is associated with an increased mortality and morbidity, ${ }^{29} 30$ over a 5 year time frame our group with HFNEF has also shown an increased hospitalisation rate, most probably due to AMC-triggered diastolic dysfunction. During the observation period, $32 \%$ (7/22) of patients with HFNEF were hospitalised with heart failure symptomatology with a median length of stay of 7.0 (4.0-8.0) days in hospital during readmission, whereas only one patient without HFNEF was in hospital for 4 days. This is important to note since the morbidity and mortality in patients with severe HFNEF have also been described as being similar to those of patients with heart failure and reduced $E^{31-33}$

In conclusion, we present evidence for the first time that after a severe form of AMC, $\sim 49 \%$ of the patients still complain about cardiac failure symptoms despite preserved EF. This group of patients developed isolated diastolic dysfunction. Further studies have to prove whether our finding can also be translated to milder forms of acute myocarditis. Our data call for mandatory monitoring and treatment of diastolic dysfunction in all patients from the onset of AMC, because long-term pathological development cannot be excluded. The essential result of our study is that the development of a HFNEF can be triggered by suffering from an AMC, and that this is independent of classical risk factors for this disease.

Funding This study was supported by a Deutsche Forschungsgemeinschaft (DFG) grant to the SFB Transregio 19 (A2, B5, Z1).

Competing interests None.

Ethics approval This study was conducted with the approval of the Deutsche Forschungsgemenschaft through SFB/Transregio 19.

Provenance and peer review Not commissioned; externally peer reviewed.

\section{REFERENCES}

1. Bowles NE, Ni J, Kearney DL, et al. Detection of viruses in myocardial tissues by polymerase chain reaction. Evidence of adenovirus as a common cause of myocarditis in children and adults. J Am Coll Cardiol 2003:42:466-72.

2. Kühl U, Pauschinger $M$, Seeberg $M$, et al. Viral persistence in the myocardium is associated with progressive cardiac dysfunction. Circulation 2005:112:1965-70.

3. D'Ambrosio A, Patti G, Manzoli A, et al. The fate of acute myocarditis between spontaneous improvement and evolution to dilated cardiomyopathy: a review. Heart 2001:85:499-504.

4. Blauwet LA, Cooper LT. Myocarditis. Prog Cardiovasc Dis 2010;52:274-88.

5. Liu PP, Mason JW. Advances in the understanding of myocarditis. Circulation 2001:104:1076-82.

6. Frustaci A, Pieroni M, Chimenti C. The role of endomyocardial biopsy in the diagnosis of cardiomyopathies. Ital Heart $J$ 2002;3:348-53.

7. Ardehlali H, Oasim A, Cappola T, et al. Endomyocardial biopsy plays a role in diagnosing patients with unexplained cardiomyopathy. Am Heart J 2004;147:919-23.

8. Cooper LT, Baughman KL, Feldman AM, et al; American College of Cardiology European Society of Cardiology. The role of endomyocardial biopsy in the management of cardiovascular disease: a scientific statement from the American Heart Association, the American College of Cardiology, and the European Society of Cardiology. Circulation 2007;116:2216-33.

9. Kasner M, Westermann D, Steendijk P, et al. Utility of Doppler echocardiography and tissue Doppler imaging in the estimation of diastolic function in heart failure with normal ejection fraction: a comparative Doppler-conductance catheterization study. Circulation 2007;116:637-47.

10. Paulus WJ, Tschope C, Sanderson JE, et al. How to diagnose diastolic heart failure: a consensus statement on the diagnosis of heart failure with normal left ventricular 
ejection fraction by the Heart Failure and Echocardiography Associations of the European Society of Cardiology. Eur Heart J 2007;28:2539-50.

11. Swedberg K, Cleland J, Dargie $\mathrm{H}$, et al. Task Force for the Diagnosis and Treatment of Chronic Heart Failure of the European Society of Cardiology. Guidelines for the diagnosis and treatment of chronic heart failure: executive summary (update 2005): The Task Force for the Diagnosis and Treatment of Chronic Heart Failure of the European Society of Cardiology. Eur Heart J 2005;26:1115-40.

12. Kühl U, Noutsias M, Seeberg B, et al. Immunohistological evidence for a chronic intramyocardial inflammatory process in dilated cardiomyopathy. Heart 1996; 75:295-300

13. Aretz HT, Billingham ME, Edwards WD, et al. Myocarditis: a histopathological definition and classification. Am J Cardiovasc Pathol 1987:1:3-14.

14. Escher F, Kuhl U, Sabi T, et al. Immunohistological detection of parvovirus B19 capsid proteins in endomyocardial biopsies from dilated cardiomyopathy patients. Med Sci Monit 2008;14:333-8.

15. Noutsias $\mathbf{M}$, Pauschinger $\mathbf{M}$, Ostermann $\mathrm{K}$, et al. Digital image analysis system for quantification of infiltrates and cell adhesion molecules in inflammatory cardiomyopathy. Med Sci Monit 2002;8:59-71.

16. Gutberlet M, Spors B, Thoma T, et al. Suspected chronic myocarditis at cardiac MR: diagnostic accuracy and association with immunohistologically detected inflammation and viral persistence. Radiology 2008;246:401-9.

17. McCarthy RE, Boehmer JP, Hruban RH, et al. Long-term outcome of fulminant myocarditis as compared with acute (nonfulminant) myocarditis. $N$ Engl J Med 2000;342:690-5

18. Caforio AL, Calabrese F, Angelini A, et al. A prospective study of biopsy-proven myocarditis: prognostic relevance of clinical and aetiopathogenetic features at diagnosis. Eur Heart J 2007;8:326-33

19. Tschöpe C, Kasner M, Westermann D, et al. The role of NT-proBNP in the diagnostics of isolated diastolic dysfunction: correlation with echocardiographic and invasive measurements. Eur Heart J 2005;26:2277-84.

20. Grewal J, McKelvie RS, Persson $\mathrm{H}$, et al. Usefulness of $\mathrm{N}$-terminal pro-brain natriuretic peptide and brain natriuretic peptide to predict cardiovascular outcomes in patients with heart failure and preserved left ventricular ejection fraction. Am J Cardiol 2008:102:733-7.
21. Watanabe S, Shite J, Takaoka H, et al. Myocardial stiffness is an important determinant of the plasma brain natriuretic peptide concentration in patients with both diastolic and systolic heart failure. Eur Heart J 2006;27:832-8.

22. Fischer M, Beassler A, Hense HW, et al. Prevalence of left ventricular diastolic dysfunction in the community: results from a Doppler echocardiographic-based survey of a population sample. Eur Heart J 2003:24:320-8.

23. Klapholz M, Maurer M, Lowe AM, et al. New York Heart Failure Consortium. Hospitalization for heart failure in the presence of normal left ventricular ejection fraction: results of New York Heart Failure Registry. J Am Coll Cardio/ 2004:43:1432-8.

24. Kindermann I, Kindermann M, Kandolf R, et al. Predictors of outcome of patients with suspected myocarditis. Circulation 2008;118:639-48.

25. Heymans S, Pauschinger M, De Palma A, et al. Inhibition of urokinase-type plasminogen activator or matrix metalloproteinases prevents cardiac injury and dysfunction during viral myocarditis. Circulation 2006:114:565-73.

26. Kawaguchi M, Hay I, Fetics B, et al. Combined ventricular systolic and arterial stiffening in patients with heart failure and preserved ejection fraction: implications for systolic and diastolic reserve limitations. Circulation 2003;107:714-20.

27. Zile MR, Brutsaert DL. New concepts in diastolic dysfunction and diastolic hear failure, part II: causal mechanisms and treatment. Circulation 2002;105:1503-8.

28. van Heerebeek L, Hamdani $\mathrm{N}$, Handoko ML, et al. Diastolic stiffness of the failing diabetic heart: importance of fibrosis, advanced glycation end products, and myocyte resting tension. Circulation 2008;117:43-51.

29. Owan TE, Hodge D0, Herges RM, et al. Trends in prevalence and outcome of heart failure with preserved ejection fraction. N Engl J Med 2006;355:251-9.

30. Varadarajan P, Pai RG. Prognosis of congestive heart failure in patients with norma versus reduced ejection fractions: results from a cohort of 2,258 hospitalized patients. J Card Fail 2003;9:107-12.

31. Vasan RS, Benjamin EJ, Levy D. Prevalence, clinical features and prognosis of diastolic heart failure: an epidemiologic perspective. J Am Coll Cardiol 1995;26:1565-74.

32. Bhatia RS, Tu JV, Lee DS, et al. Outcome of heart failure with preserved ejection fraction in a population-based study. N Engl J Med 2006:355:260-9.

33. Schaefer A, Meyer GP, Fuchs M, et al. Impact of intracoronary bone marrow cell transfer on diastolic function in patients after acute myocardial infarction: results from the BOOST trial. Eur Heart J 2006:27:929-35.

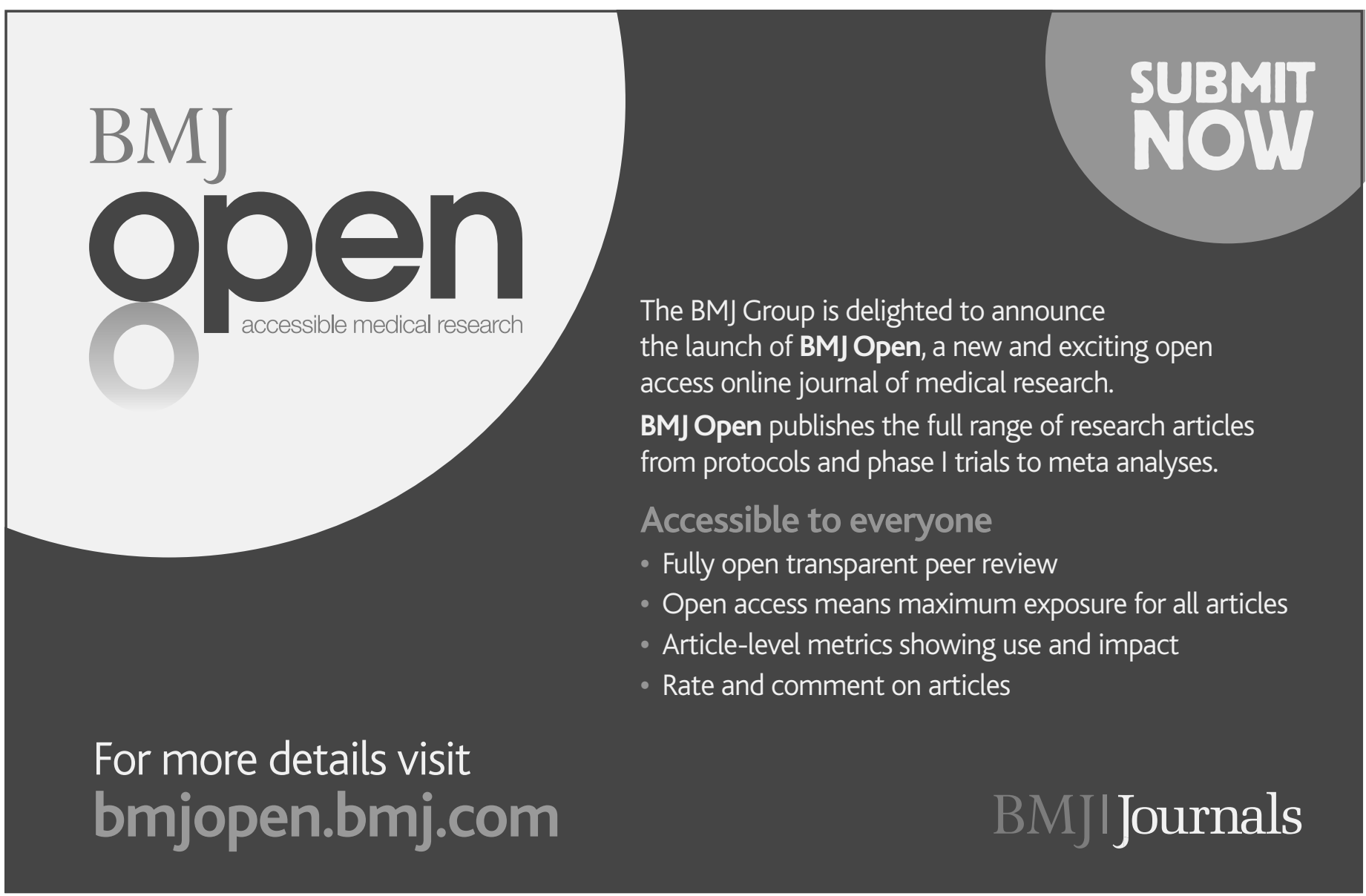

